children-which included all those with adverse criteriabut grave problems beset the families and upset normal siblings. ${ }^{2}$

Having defined his adverse prognostic criteria, Lorber tested them against the patients admitted to the Sheffield unit during the last ten years. The results confirm their validity and raise the question of the moral justification for treating all patients with spina bifida cystica without some form of selection. As has been mentioned, all the patients with adverse criteria were severely disabled, but $59 \%$ died despite maximum efforts at treatment. On the other hand the survival of those with open myelomeningoceles but without the adverse criteria was $84 \%$, and their quality of survival was very much better. Half of them were of normal intelligence compared with only one-fifth of those with adverse criteria, who also required many more operations for their hydrocephalus and urinary and orthopaedic disturbances.

The problem of what treatment should be offered was presented in a particularly acute form at Hull recently. ${ }^{3}$ In that case the parents, who disagreed with their medical advisers, were overridden by the juvenile court on the application of the social services committee. Many people have regarded the circumstances of this case as disquieting.

It may be thought wrong to keep patients alive merely because it is technically possible to do so. A study by P. P. Rickham and T. Mawdsley ${ }^{4}$ shows that a miserable survival without treatment is not common, for they found only two children were alive after six months among 57 untreated cases of spina bifida cystica. Lorber has provided valuable evidence on which to base a system of selection. But there can be no universally applicable formula; every case must be regarded as an individual problem.

\footnotetext{
1 Lorber, J., Archives of Disease in Childhood, 1972, 47, 854.

2 Hare, E. H., Laurence, K. M., Payne, H., and Rawnsley, K., British Medical fournal, 1966, 2, 757

British Medical fournal, 1972, 4, 567.

- Rickham, P. P., and Mawdsley, T., Developmental Medicine and Child Neurology, 1966, 8, Supplement 1i, p. 20.
}

\section{Obesity and Coronary Heart Disease}

A particular type of person is often depicted as being specially liable to coronary artery disease. Characteristically he is an obese cigarette smoker, employed in a sedentary but stressful occupation, which affords him little physical activity, and he may have a family history of cardiovascular disease. On examination he may be hypertensive, have a corneal arcus, xanthelesma, and xanthomata, and the laboratory may detect hypercholesterolaemia, lipoprotein abnormalities, or diabetes mellitus.

How accurate is this profile? It is agreed that hypercholesterolaemia, cigarette smoking, hypertension, and diabetes are real risks, ${ }^{1-5}$ and it is generally accepted that obesity also promotes the development of coronary artery disease. ${ }^{7}$ This belief is based on detailed follow-up studies and on actuarial reports of insurance companies ${ }^{8-11}$ suggesting that there is a continuous direct relationship between body weight and coronary disease. However, the validity of this assumption has been questioned. 3467

Opinions are bound to conflict because we know so little about obesity. It lacks any absolute definition, and often no distinction is made between obesity and overweight. ${ }^{12}$ There is lack of agreement on how it can best be measured, and there are many methods of expressing body weight. There is even doubt about the accuracy of a measure which must estimate bone, muscle, and body shape in addition to obesity. ${ }^{710}$ Skinfold thickness is possibly a more accurate indicator than body weight of actual fatness, but measurements of it have not been adopted as a routine. ${ }^{12} 13$ Insurance companies' tables of desirable weight are based on the average weights of insurance applicants of given sex and height, aged about 25 , and arbitrarily divided into types of body frame to allow for skeletal variation. These tables have been much criticized. 467 Finally, epidemiological studies are subject to statistical difficulties, especially in evaluating the significance of obesity in isolation from other risk factors such as hypertension, hypercholesterolaemia, and cigarette smoking.

In an attempt to resolve some of these problems Ancel Keys and his colleagues have analysed the relationship of relative weight and skinfold thickness to the five-year incidence of coronary heart disease in 11,400 men aged 4059.14 There were three geographical groupings-northern European (Finland and the Netherlands), southern European (Italy and Yugoslavia), and U.S. railroad employees. Two measures of relative body weight were used, and skinfold thickness, which is independent of height, was also measured. A number of interesting findings emerge from this study. On the basis of relative body weight a greater proportion of the Americans (29.5\%) and southern Europeans $(23.1 \%)$ were overweight than were the northern Europeans $(13 \%)$. At first it appeared that those who were overweight (as judged by relative body weight) and those who were obese (as judged by measurement of skinfold thickness) among the Americans and southern Europeans were more liable than average to coronary artery disease, but this association did not approach significance in relation to death from this disease or to definite myocardial infarction. Multivariate analysis of the data showed that neither relative weight nor obesity was in itself a statistically significant risk in relation to future coronary heart disease when age, blood pressure, serum cholesterol, and smoking were comparable. But, as expected, relative weight and fatness correlated with blood pressure and serum cholesterol, and there was an inverse correlation with smoking habit, the smokers being thinner and lighter than the non-smokers.

It may be true, as these findings suggest, that obesity itself does not necessarily increase the risk of coronary artery disease. How then, should the physician advise the overweight patient? The answer would seem to be that, though overweight may not be an independent risk factor, there are many compelling grounds for weight reduction. In the first place, a diseased heart should not be subjected to the unnecessary strain of moving an overweight body. Secondly, obesity is associated with the development of hypertension, hyperlipidaemia, and diabetes, ${ }^{19}$ all of which are aetiologically important in coronary heart disease, ${ }^{1-5}$ and weight reduction should be the first (and is often the most successful) treatment for these conditions. Apart from the cardiovascular consequences, obesity is apt to be associated with sedentary habits and skeletal and pulmonary complications. 9

1 Doyle, J. T., Dawber, T. R., Kannel, W. B., Kinch, S. H., and Kahn, H. A., fournal of the American Medical Association, 1964, 190,886 . 
2 Rosenman, R. H., et al., fourmal of Chronic Diseases, 1970, 23, 173.

3 Report of Inter-Society Commision for Heart Disease Resources, Circulation, 1970, 42, A55.

Keys, A., Circulation, 1970, 41, Suppl. 1.

5 Kann:1, W. B., Castelli, W. P., Gordon, T., and McNamara, P. M., Annals of Internal Medicine, 1971, 74, 1 .

6 Keys, A, and Blackburn, H., Progress in Cardiovascular Diseases, $1963,6,14$.

7 Seltzer, C. C., New Eneland fournal of Medicine, 1966, 274, 254.

8 Society of Actuaries, Build and Blood Pressure Study, Vol. 1. Chicago, 1959.

- Marks, $H$. 36, 296. Kannel, W. B., Le Bauer, E. J., Dawber, T. R., and McNamara, P. M., Circulation, 1967, 35, 734.

11 Truett, J., Cornfield, J., and Kannel, W., foumal of Chronic Diseases, $1967,20,511$.

12 Sanders, K., Lancet, 1959, 2, 432.

13 Paul, O. et al., Circulation, 1963, 28, 20.

14 Keys, A. et al., Annals of Internal Medicine, 1972, 77, 15.

\section{Early Diagnosis of Nephroblastoma}

A renal tumour occurring in an infant or child is usually a nephroblastoma (Wilms's tumour). The untreated disease ends inevitably in death-unlike the neuroblastoma, which, panticularly in the first year of life, may spontaneously regress or change to ganglioneuroma. Early diagnosis of nephroblastoma remains vital to the survival of the infant or child.

As $80 \%$ of nephroblastomas occur in children under the age of 5 years and $50 \%$ under the age of 3, the symptomatology is not clearly expressed. Vague abdominal pain, irritability, and malaise are common presenting complaints. A mass in the abdomen is the most important sign. This mass is either noticed by the parents when bathing or dressing the child or is found by the doctor, sometimes during an examination for an unrelated reason. Two other signs which may occur are haematuria and fever. Haematuria is uncommon but is important, as its misdiagnosis has been shown to be the main cause of delay after medical advice has been sought. ${ }^{2}$ Unless the child with haematuria has a clear cause for it, an intravenous pyelogram should be performed as soon as possible. The lack of danger of an intravenous pyelogram to patients with haematuria from other causes such as nephritis is now accepted. This examination should be done early, and then appropriate treatment can be begun. Fever is another sign which may be present early in about $20 \%$ of patients with nephroblastoma. The cause of the fever is not entirely clear. It is not always related to necrosis in the tumour, nor is there evidence of infection in the adjacent compressed renal tissue. Undefined fever may precede other complaints. Careful abdominal palpation of all infants and children is thus necessary to detect nephroblastoma at the earliest possible time.

The family incidence of nephroblastoma, particularly in twins and in other sibs, has been well documented. Comparable evidence of the disease occurring in successive generations has not been available. T. Strøm ${ }^{3}$ reported a nephroblastoma family, but this report had histological confirmation of the diagnosis in only one of the five probable cases. Two recent reports from the United States helped substantiate the famililial incidence of the disease..$^{45}$

W. T. Brown and colleagues 4 described a family in whom the grandfather had a right nephrectomy in 1915 for a presumed nephroblastoma. This man had four offspring. The eldest girl has had no renal tumours and her two sons are well. The second girl had a confirmed right nephroblastoma excised at 14 months of age but then developed left nephroblastoma at 7 years and died. The third female had right nephrectomy for confirmed nephroblastoma when 6 months old. Her daughter also had a right nephroblastoma diagnosed at six months. The fourth pregnancy was terminated at the fourth month and the fetus was stated to have bilateral renal tumours, but no histological examination was made of the kidneys. R. L. Kaufmans and colleagues have reported on father and son with left and right nephroblastoma respectively.

The penetrance of the genetic factors appears to be rather low in general, though in some families it may be high. With the increasing survival of patients treated for nephroblastoma the occurrence of the tumour in successive generations can be expected more often. The full significance of the genetic factors should become clearer over the next decade. Meantime, it is important that the children of parents who had nephroblastoma should have regular examination to detect early signs of it. Close surveillance would seem advisable until the children have reached school age.

One further small group of patients previously considered to have nephroblastoma has been identified in recent years. 7 These kidney tumours presenting in the neonatal period are now distinguished from nephroblastoma: several names have been suggested, and two of the more acceptable are fibrosarcoma and congenital mesonephric nephroma. The importance of identifying this group is to prevent the infant from receiving radio therapy and chemotherapy. Infants with such tumours have died as a result of radiotherapy and chemotherapy but not with metastatic spread of the disease. Nophrectomy is curative. The tumours are uncommon, and it is likely to be some time before there is evidence to indicate if they fit into the genetic pattern of nephroblastoma or if they are also distinct in that aspect as well as in the clinical course.

\footnotetext{
1 Williams, D. I., and Young, D. G., Practitioner, 1968, 200, 678.

2 Ledlie, E. M., Mynors, L. S., Draper, G. J., and Gorbach, P. D., British Medical fournal, 1970, 4, 195.

Strom, T., Acta Paediatrica Scandinavica, 1957, 46, 601.

- Brown, W. T., Puranik, S. R., Altman, D. H., and Hardin, H. C., Surgery, $1972,72,756$

5 Kaufman, R. L., Vietti, T. J., and Wabner, C. I., Lancet, 1973, 1, 43.

- Young, D. G., and Williams, D. I., British fournal of Hospital Medicine $1969,2,74$.

7 Favara, B. E., Johnson, W., and Ito, J., Cancer, 1968, 22, 845.
}

\section{Antibiotic Levels in Tissue Fluid}

The distribution of antibiotics and other antibacterial drugs in the body is clearly an important factor in therapeutic success. It has been studied in many ways, but for most such drugs the main information available consists of "blood levels," and schemes of dosage have been largely based on these. Except in septicaemia the concentration in the blood itself is not directly important: what matters is that attained, and how long it is maintained, in the infected tissues into which the drug diffuses. Here much will depend on the nature of the lesion, and in particular on whether there is tissue breakdown. The early studies at Oxford ${ }^{1}$ showed that penicillin persisted in the pus of suppurating wounds for eight to twelve hours after an intramuscular dose which produced an effective blood level for less than four hours. This 